\title{
MICROSPOROSE CANINA E HUMANA - UM RELATO DE CASO ZOONÓTICO
}

\author{
WALLER, Stefanie Bressan ${ }^{1}$ \\ GOMES, Angelita dos Reis ${ }^{2}$; \\ CABANA, Ângela Leitzke ${ }^{2}$; \\ FARIA, Renata de Osório ${ }^{3}$; \\ MEIRELES, Mário Carlos Araújo ${ }^{4}$; \\ MELLO, João Roberto Braga de ${ }^{5}$.
}

\footnotetext{
${ }^{1}$ Mestranda do Programa de Pós-Graduação em Ciências Veterinárias - UFRGS, ${ }^{2}$ MSc., Doutoranda do Programa de Pós-Graduação em Veterinária - UFPEL, ${ }^{3}$ Dra. Prof. Adjunta de Doenças Infecciosas dos Animais Domésticos FAVET/UFPEL, ${ }^{4}$ Dr. Prof. Associado de Doenças Infecciosas dos Animais Domésticos - FAVET/UFPEL, ${ }^{5}$ Dr. Prof. Associado, Departamento de Farmacologia, Instituto de Ciências Básicas da Saúde - UFRGS.
}

\section{RESUMO}

$\mathrm{M}$ icrosporose é uma infeç̧ão fúngica cutânea causada por dermatófito do gênero Microsporum, que apresenta potencial zoonótico. Um canino, fêmea, da raça Poodle, com 2 anos e 6 meses de idade, foi encaminhado para exame clínico com queixa principal de múltiplas lesões cutâneas alopécicas, eritematosas e pruriginosas, com cerca de $1 \mathrm{~cm}$ de diâmetro, circular, e pouca secreção purulenta, localizadas no plano nasal, dorsal torácico e membros anterior esquerdo e posterior direito proximais. Verificou-se que a proprietária do animal também apresentava lesões cutâneas similares. Foi realizada a coleta de material clínico no animal, através do arrancamento de pelos das bordas das lesões e swab estéril, e na proprietária foi utilizada a técnica do carpete e raspado cutâneo. No exame direto dos pelos do canino, não foi evidenciada presença de estruturas fúngicas. $\mathrm{Na}$ cultura fúngica do material clínico animal e humano, observou-se crescimento do fungo Microsporum canis. O diagnóstico foi realizado com base no histórico, anamnese e exame complementar micológico. Conclui-se a importância da realização de exames complementares laboratoriais na presença de lesões suspeitas, uma vez que a dermatofitose é uma zoonose de grande importância em saúde pública, pelo seu dispendioso e longo tratamento, que deve ser realizado tanto em animais como humanos e no ambiente afetado, muitas vezes persistindo mesmo depois da obtenção da cura clínica.

Palavras-chave: Dermatofitose. Zoonose. Micose. Cão. Microsporum canis. 


\section{INTRODUÇÃO}

A dermatofitose é uma micose cutânea causada por aproximadamente 30 espécies de fungos pertencentes aos gêneros Microsporum, Trichophyton e Epidermophyton e que apresentam afinidade por substratos queratinizados da pele e seus anexos, como unhas e pelos, sendo transmitidos através do contato direto dos esporos com o hospedeiro suscetível (CAFARCHIA et al., 2006; CHERMETTE et al., 2008; MORIELLO, 2004; NEVES et al., 2011; RINALDI, 2000).

A infecção pelos dermatófitos é possível por apresentarem capacidade de invadir o extrato córneo e o óstio folicular e, dependendo do estado imunológico do hospedeiro, o tamanho e a duração das lesões provocadas podem variar em maior ou menor grau. Frequentemente, observam-se alopecia circular demarcada com inflamação ativa na periferia, podendo apresentar crostas na região central com prurido (CHERMETTE et al., 2008).

Dentre as espécies dermatofíticas, Microsporum canis apresenta elevada incidência em pequenos animais e frequentemente está envolvido em casos zoonóticos (REIS-GOMES, 2012; REIS-GOMES et al., 2012). A transmissão pode ocorrer através do contato direto com pelos, pele, e/ou crostas contaminadas de um portador sintomático ou assintomático. Em se tratando de animais de companhia, os felinos são considerados o principal reservatório de M. canis. A infecção por contato indireto pode ocorrer através de fômites e ambiente contaminado (MEIRELES; NASCENTE, 2009; REIS-GOMES et al., 2012).

O presente estudo teve como objetivo relatar a ocorrência de dermatofitose por $M$. canis em uma cadela da raça Poodle e na sua proprietária.

\section{RELATO DE CASO}

Um canino, fêmea, da raça Poodle, de 2 anos e 6 meses de idade, foi atendida em uma clínica veterinária particular com queixa principal de presença de múltiplas lesões cutâneas generalizadas, compreendendo as áreas da extensão do plano nasal, face lateral dos membros anteriores e posteriores e região torácica lateral direita. Ao exame clínico, observaram-se lesões cutâneas alopécicas, pruriginosas, eritematosas, de aspecto circular, 
com cerca de $1 \mathrm{~cm}$ de diâmetro e presença de alto relevo central com pouca secreção purulenta. Não foram observadas outras alterações clínicas. Ainda, observou-se que a proprietária apresentava lesões similares, localizadas na face e principalmente nas regiões dos braços e antebraços (Figura 1).

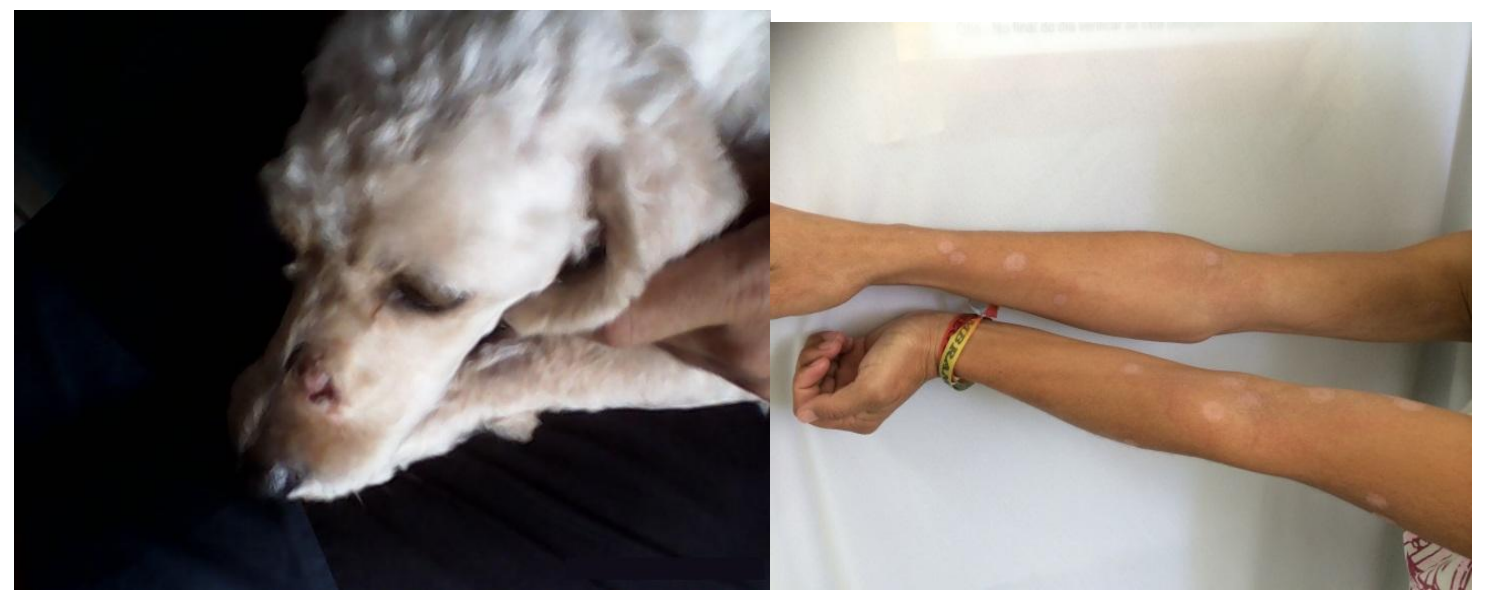

Figura 1 - Lesões cutâneas, circulares, alopécicas e pruriginosas, localizadas no plano nasal de uma cadela da raça Poodle (esquerda) e nos antebraços e braços da proprietária (direita).

Com base nos sinais clínicos do animal e da proprietária, a suspeita clínica foi de dermatofitose, sendo realizada antissepsia local com álcool $70^{\circ} \mathrm{GL}$ para posterior coleta de material biológico através de swab estéril, raspado cutâneo com lâmina de bisturi estéril e arrancamento de pelos presentes nas bordas das lesões do animal. Na proprietária, foi realizada coleta de material por meio do uso da técnica do carpete e raspado cutâneo após antissepsia prévia com álcool $70^{\circ} \mathrm{GL}$.

As amostras clínicas foram processadas individualmente para exame micológico, sendo realizado o exame direto dos pelos e crostas através da clarificação em Hidróxido de Potássio a 20\% $\left(\right.$ Synth $^{\circledR}$, Labsynth, Diadema, SP) e análise microscópica para avaliação da presença de parasitismo endothrix ou ectothrix. Posteriormente, foram realizadas semeaduras das amostras em meios de cultura ágar Sabouraud dextrose (Sabouraud Dextrose Agar ${ }^{\circledR}$, Neogen Corporation-Lansing, Michigan, USA) acrescido de cloranfenicol e ciclohexemida (Mycobiotic Agar ${ }^{\circledR}$, Neogen Corporation-Lansing, Michigan, USA) e incubadas a $25{ }^{\circ} \mathrm{C}$ por dez dias com observação diária. O exame direto das colônias foi realizado através da coleta de uma alçada do crescimento fúngico e transferida para uma lâmina de vidro 
estéril contendo uma gota de Azul de Lactofenol (Azul de Algodão CB Certificado, Inlab, Alamar Tecno-Científica, São Paulo, SP), seguida da colocação de uma lamínula, e observação em microscópio óptico em aumento 100 e 400x.

\section{RESULTADOS E DISCUSSÃO}

No exame direto dos pelos, não foram observadas estruturas fúngicas. Em cinco dias de incubação, observou-se crescimento fúngico na amostra clínica canina e na amostra humana. As colônias macroscópicas foram caracterizadas com textura aveludada e algodonosa, de coloração branca no verso da placa de Petri e de coloração amarelo-alaranjada no reverso (Figuras 2 e 3). A análise microscópica em Azul de Lactofenol foi realizada diariamente para obtenção de uma melhor visualização das estruturas fúngicas de reprodução, a qual foi possível a partir do 10 dia de incubação. Observaram-se numerosas hifas septadas e hialinas com presença de macroconídios de formato fusiforme, parede espessa e rugosa, com divisão de 8 a 12 septos, assim como a presença de microconídios (DVORAK; OTCENASEK, 1969; REIS-GOMES et al., 2012), cujo diagnóstico micológico foi de $M$. canis (Figura 4).

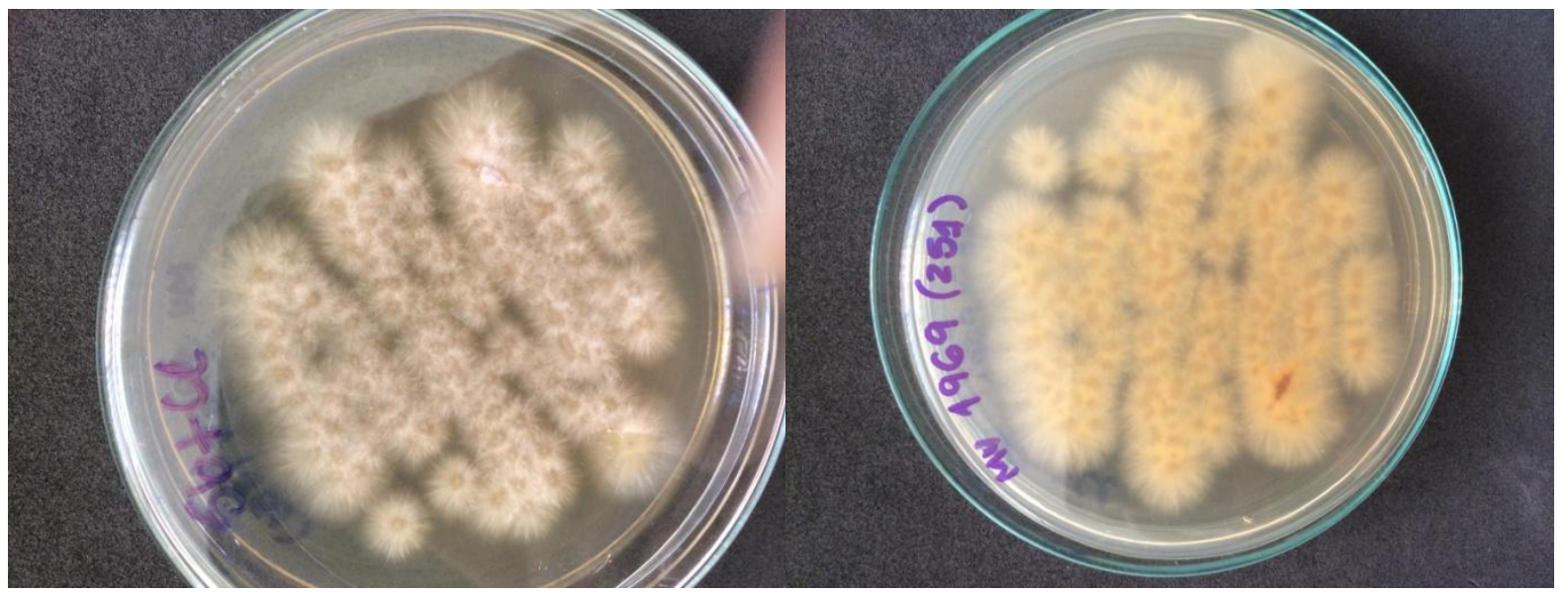

Figura 2 - Macroscopia fúngica em ágar Sabouraud dextrose com cloranfenicol, de material biológico cutâneo de canino, evidenciando crescimento de colônias aveludadas, com halos dispersos em sentido radial e coloração branca a amarelada no verso (esquerda) e alaranjada no reverso (direita), característico de $M$. canis. 


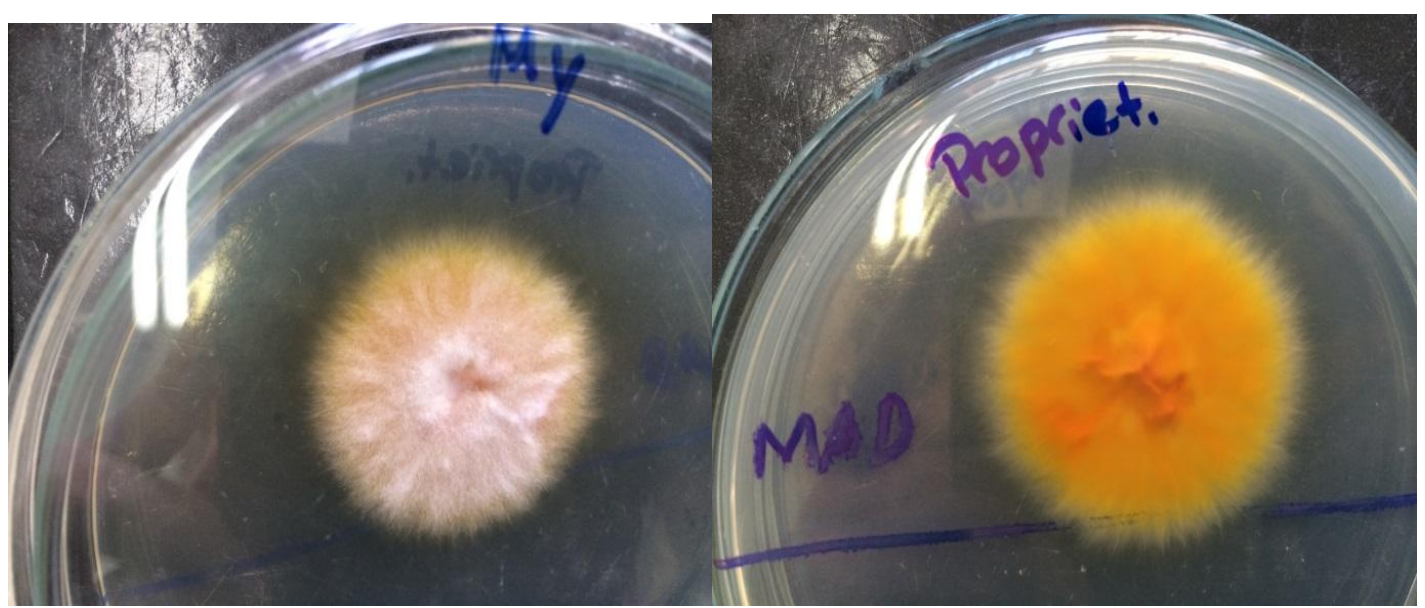

Figura 3 - Macroscopia fúngica em meio ágar Sabouraud-dextrose acrescido de cloranfenicol e ciclohexemida, de material biológico oriundo de humano, demonstrando no verso (esquerda) crescimento de colônia aveludada, algodonosa e com halos radiais, e no reverso (direita) coloração alaranjada, característico de $M$. canis.

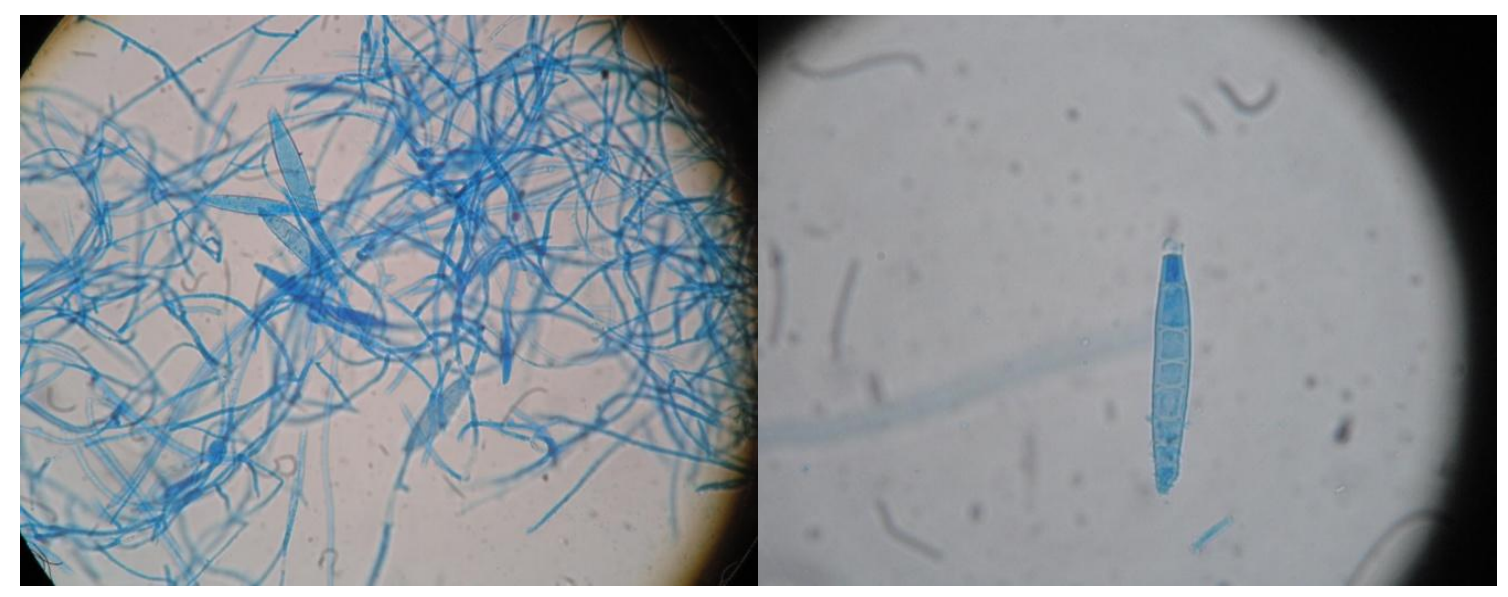

Figura 4 - Microscopia em coloração de azul de Lactofenol, utilizando aumento de 40x. Amostras de isolado fúngico oriundo de canino (esquerda) e de humano (direita). Evidenciam-se a presença de numerosas hifas septadas e hialinas com presença de macroconídios fusiformes contendo de oito a doze septos, característicos de M. canis.

A dermatofitose é uma enfermidade infecciosa de difícil controle e erradicação, tanto no homem quanto nos animais domésticos (REIS-GOMES et al., 2012). Cães e gatos são conhecidos como reservatórios potenciais de diversas patologias humanas, os quais favorecem a transmissão de patógenos por dividirem o mesmo "nicho urbano" (CHINELLI et al., 2003; PINHEIRO et al., 1997).

No presente relato, $M$. canis foi isolado do tegumento cutâneo do animal, sendo esta espécie dermatofítica considerada a mais isolada entre os estudos de dermatofitose em 
caninos (BAGCIGIL et al., 2010; BIN et al., 2010; BRILHANTE, 2005; CAFARCHIA et al., 2004; MORIELLO, 2004; PALUMBO et al., 2010; PRADO et al., 2008; SEKER; DOGAN, 2011). Em humanos, essa espécie é a segunda mais frequente no Brasil (RUIZ; ZAITZ, 2001).

Por ser um fungo zoofílico de distribuição mundial, $M$. canis é frequentemente encontrado na pelagem de caninos, felinos e equinos assintomáticos (SHARMA et al., 2007).

As infecções humanas por dermatófitos zoofílicos são esporádicas e estão diretamente associadas a animais infectados (PINHEIRO et al., 1997), o que torna essencial a adoção de exames complementares para o diagnóstico micológico. Em geral, as lesões cutâneas da dermatofitose em caninos tendem a ser distribuídas de forma generalizada, principalmente nas regiões cefálica, tronco, abdômen e membros (NEVES et al., 2011; PALUMBO et al., 2010), conforme observado no presente relato, entretanto, Balda et al. (2004) citam que a região cervical é a mais acometida na dermatofitose canina. Os sinais clínicos observados foram alopecia, eritema e prurido, estando em concordância com os relatos de literatura (BALDA et al., 2004; NEVES et al., 2011; PALUMBO et al., 2010). Crostas, pápulas e colarete epidérmico também podem ser observados em caninos com dermatofitose (BALDA et al., 2004; CAFARCHIA et al., 2004; NEVES et al., 2011).

Embora tenham sido observadas lesões clássicas da dermatofitose no animal, correspondendo a suspeita clínica do diagnóstico, reforçamos as recomendações de Chermette et al. (2008) quanto à ocorrência de lesões dermatofíticas atípicas, devido ao grau de inflamação presente, o que pode alterar o aspecto clássico das lesões e dificultar a suspeita clínica. Reis-Gomes et al. (2012), reforçam a utilização do diagnóstico laboratorial nos casos suspeitos de dermatofitose, pois somente a avaliação clínica das lesões pode levar ao erro diagnóstico. Assim, exame direto dos pelos e crostas, cultura fúngica, lâmpada de Wood e biópsia cutânea são consideradas técnicas diagnósticas para dermatofitose em associação ao histórico e exame clínico (REIS-GOMES et al., 2012).

Dentre as medidas de controle e profilaxia, recomenda-se o isolamento de animais doentes e a desinfecção de pisos, instalações e utensílios com produtos químicos, como hipoclorito de sódio a $10 \%$ e soda cáustica a $5 \%$, a fim de controlar a cadeia de transmissão de dermatófitos, uma vez que os artroconídios de $M$. canis e outros dermatófitos podem 
permanecer por até 18 meses no ambiente. Entretanto, o isolamento e a desinfecção nem sempre são utilizados, pois é comum a existência de animais portadores assintomáticos (MEIRELES; NASCENTE, 2009).

A dermatofitose por $M$. canis merece destaque por seu potencial de transmissão zoonótico, como observado no relato clínico, uma vez que a convivência entre humanos e animais de companhia cada vez aumenta mais, facilitando a transmissão de patógenos entre espécies diferentes, o que reforça a adoção de exames complementares e medidas de controle adequadas para prevenção da enfermidade (CAFARCHIA et al. 2006; CHERMETTE et al., 2008).

\section{CONCLUSÃO}

A observação de lesões cutâneas similares na proprietária e no seu animal de estimação, levou à suspeita clínica de dermatofitose, confirmada através do exame micológico que identificou Microsporum canis como o agente da enfermidade. A utilização de exames complementares associados à anamnese, histórico e exame clínico, é recomendada e fundamental para o diagnóstico da doença, uma micose de elevado potencial zoonótico, de grande importância para a saúde pública e que exige cuidados específicos para seu tratamento e controle.

\section{CANINE AND HUMAN MICROSPOROSIS - A ZOONOTIC CASE REPORT}

\section{ABSTRACT}

M icrosporosis is a fungal skin infection caused by dermatophytes of the Microsporum genus, which have zoonotic potential. A canine, female, Poodle, with 2 years and 6 months of age, was referred for clinical examination because it had multiple alopecic, erythematous and pruritic circular skin lesions, with approximately 1 $\mathrm{cm}$ in diameter and purulent aspect. The lesions were located in the nasal plane, dorsally in the thorax and proximally in the anterior left and posterior right limbs. It was found out that the dog's owner also had similar skin lesions. So, collection of clinical material from the animal was performed through sterile swabs and the removal of fur from the edges around 
the lesions. The carpet technique and skin scraping were employed to collect samples from the owner of the dog. On the direct examination of canine fur, fungal structures were not evidenced. In the fungal cultures of animal and human clinical materials, there was the growth of Microsporum canis. The diagnosis was made based on the clinical history and the complementary mycological examination. Complementary laboratory tests in the presence of suspected lesions are essential, because dermatophytosis is a zoonotic disease of great public health importance. Furthermore, long and expensive treatments must be performed in animals, humans and environment, where the fungus often persists even after achieving clinical cure.

Keywords: Dermatophytosis. Zoonosis. Mycosis. Dog. Microsporum canis.

\section{MICROSPOROSIS CANINA Y HUMANA - RELATO DE UN CASO ZOONÓTICO}

\section{RESUMEN}

$\mathrm{M}$ icrosporosis es una infección fúngica de la piel causada por dermatofitos del género Microsporum, que tienen potencial zoonótico. Un canino, hembra, de la raza Poodle, con 2 años y 6 meses de edad, fue remitida a la consulta clínica porque tenía múltiples lesiones cutáneas alopecicas, eritema y lesiones cutáneas pruriginosas, con aproximadamente $1 \mathrm{~cm}$ de diámetro y aspecto circular, con poco pus, situado en el plano nasal, dorsal torácica, y miembros anterior derecho y posterior izquierdo proximales. Se encontró que el dueño del perro también tenía lesiones en la piel similares. Por lo tanto, la recolección de material clínico en el animal se realizó a través de la retirada de los bordes alrededor de las lesiones y esponja estéril, y en el dueño del perro se utilizó la técnica de la alfombra y el raspado de la piel. El examen directo de bordes caninos, no se puso de manifiesto estructuras fúngicas. En los cultivos de hongos de los animales y el material clínico en humanos, hubo crecimiento del Microsporum canis. El diagnóstico se realiza con base en la historia clínica, anamnesis y el examen micológico. Se concluye sobre la importancia de la realización de exámenes de laboratorio complementarios cuando existen lesiones sospechosas, ya que la dermatofitosis es una zoonosis de gran importancia para la salud pública, por su largo y costoso tratamiento, que debe ser realizado tanto en los animales y los seres humanos, así como también en el medio ambiente. A pesar de esto, muchas veces la enfermedad persiste después de lograr la curación clínica.

Palabras clave: Dermatofitosis. Zoonosis. Micosis. Perro. Microsporum canis. 


\section{REFERÊNCIAS}

BAGCIGIL, A. F.; IKIS, S.; OZGUR, N. Y.; et al. Recovery of dermatophytes in pet grooming tools from veterinary clinics and pet grooming salons. Journal of Small Animal Practice, v. 51, n. 1, p. 39-42, 2010.

BALDA, A. C.; LARSSON, C. E.; OTSUKA, M.; et al. Estudo retrospectivo de casuística das dermatofitoses em cães e gatos atendidos no serviço de Dermatologia da Faculdade de Medicina Veterinária e Zootecnia da Universidade de São Paulo. Acta Scientiae Veterinariae, v. 32, n. 2, p. 133-140, 2004.

BIN, L. L. C.; GOMES, J.; BRÁZ, S. A.; et al. Comparação de métodos diagnósticos para dermatofitose em animais de companhia. Colloquium Agrariae, v. 6, n. 2, p. 46-51, 2010.

BRILHANTE, R. S. N. Caracterização fenotípica e genotípica de Microsporum canis oriundos de cães e gatos como um possível clone fúngico. Fortaleza: UECE, 2005. 82p. (Doutorado em Reprodução e Sanidade Animal), Faculdade de Veterinária, Universidade Estadual do Ceará, 2005.

CAFARCHIA, C.; ROMITO, D.; SASANELLI, M.; et al. The epidemiology of canine and feline dermatophytoses in southern Italy. Mycoses, v. 47, n. 11/12, p. 508-513, 2004.

CAFARCHIA, C.; ROMITO, D.; CAPELLI, G.; et al. Isolation of Microsporum canis from the coat of pet dogs and cats belonging to owners diagnosed with $M$. canis tea corporis. Veterinary Dermatology, v. 17, n. 5, p. 327-331, 2006.

CHERMETTE, R.; FERREIRO, L.; GUILLOT, J. Dermatophytoses in animals. Mycopathologia, v. 166, n. 5-6, p. 385-405, 2008.

CHINELLI, P. A. V.; SOFIATTI, A. A.; NUNES, R. S.; MARTINS, J. E. C. Dermatophyte agents in the city of São Paulo, from 1992 to 2002. Revista do Instituto de Medicina Tropical de São Paulo, v. 45, n. 5, p. 259-263, 2003.

DVORAK, J.; OTCENASEK, M. Mycological diagnosis of animal dermatophytosis. Oxford: Junk, 1969. 213p.

MEIRELES, M. C. A.; NASCENTE, P. S. Micologia Veterinária. Pelotas: Ed. da UFPEL, 2009. $543 p$.

MORIELLO, K. A. Treatment of dermatophytosis in dogs and cats: review of published studies. Veterinary Dermatology, v. 15, n. 2, p. 99-107, 2004.

NEVES, R. C. S. M.; CRUZ, F. A. C. S.; LIMA, S. R.; et al. Retrospectiva da dermatofitose em cães e gatos atendidos no Hospital Veterinário da Universidade Federal de Mato Grosso, nos anos de 2006 a 2008. Ciência Rural, v. 41, n. 8, p. 1405-1410, 2011. 
PALUMBO, M. I. P.; MACHADO, L. H. A.; PAES, A. C.; et al. Estudo epidemiológico das dermatofitoses em cães e gatos atendidos no serviço de dermatologia da Faculdade de Medicina Veterinária e Zootecnia da UNESP - Botucatu. Semina: Ciências Agrárias, v. 31, n. 2, p. 459-468, 2010.

PINHEIRO, A. Q.; MOREIRA, J. L. B.; SIDRIM J. J. C. Dermatofitoses no meio urbano e a coexistência do homem com cães e gatos. Revista da Sociedade Brasileira de Medicina Tropical, v. 30, n. 4, p. 287-294, 1997.

PRADO, M.; BRILHANTE, R. S.; CORDEIRO, R. A.; et al. Frequency of yeasts and dermatophytes from healthy and diseased dogs. Journal of Veterinary Diagnostic Investigation, v. 20, n. 2, p. 197-202, 2008.

REIS-GOMES, A. Estudo retrospectivo das micoses e micotoxicoses animais na região sul do Brasil. Pelotas: UFPEL, 2012. 95p. Dissertação (Mestrado em Veterinária), Faculdade de Veterinária, Universidade Federal de Pelotas, 2012.

REIS-GOMES, A.; MADRID, I. M.; MATOS, C. B.; et al. Dermatopatias fúngicas: aspectos clínicos, diagnósticos e terapêuticos. Acta Veterinaria Brasilica, v. 6, n. 4, p. 272-284, 2012.

RINALDI, M. G. Dermatophytosis: epidemiological and microbiological update. Journal American Academy of Dermatology, v. 43, n. 5, p. 120-124, 2000.

RUIZ, L. R. B.; ZAITZ, C. Dermatophytes and dermatophytosis in the city of São Paulo, from August 1996 to July 1998. Anais Brasileiro de Dermatologia e Sifilografia, v. 76, p. 391-401, 2001.

SEKER, E.; DOGAN, N. Isolation of dermatophytes from dogs and cats with suspected dermatophytosis in Western Turkey. Preventive Veterinary Medicine, v. 98, n. 1, p. 46-51, 2011.

SHARMA, R.; De HOOG, S.; PRESBER, W.; et al. A virulent genotype of Microsporum canis is responsible for the majority of human infections. Journal of Medical Mycology, v. 56, n. 1, p. 1377-1385, 2007. 